\title{
Supporting the UK National Health Service during the COVID-19 crisis from an academic perspective
}

\author{
Matthias Trost is a biochemist and mass spectrometrist working at Newcastle University. He is currently leading \\ the university's effort in supporting the National Health Service with COVID-19 screening.
}

Matthias Trost

n my professional life, I am Professor of Proteomics at Newcastle University in the UK. Since the COVID-19 pandemic led to the shutdown of university research labs in the UK, a move unprecedented in peacetime, my current daily focus is to lead efforts from within our medical faculty to support the local National Health Service (NHS) hospitals with screening for COVID19. Although I have experience with screening in drug-discovery settings, I have never performed qPCR, the current method of choice for COVID-19 detection in acute infection. So, why me?

It is not that I lack previous experience in the organization of people and events. My desire to take action in times of crisis has always shaped the course of my life and career. I have been running a successful proteomics lab for 10 years now, first in the Medical Research Council Unit in Dundee and, in the past 3 years, here at Newcastle University, following my appointment as Professor of Proteomics. When it became clear that the COVID-19 crisis would hit the UK, I was the first to approach our pro-vice chancellor to question what the faculty could do to support the NHS and Public Health England (PHE) to maximize the screening capacity in our region. This immediately became my role.

In February, most in the UK were not fully aware of how the crisis would manifest. The university was still open for business, and many were still hoping that normal life would continue. When the government began to reach out to universities for equipment to build up a national screening facility in Milton Keynes, things accelerated quickly. I reached out to colleagues to help with this undertaking. Within 2 days, we had over 650 volunteers from all levels of the university, from undergraduate students to administrators to professors. As we had specifically asked for those with qPCR experience, this number is likely a huge under-representation of those within the university ready and willing to help.

The first days were intense; I received almost 400 emails per day. The information

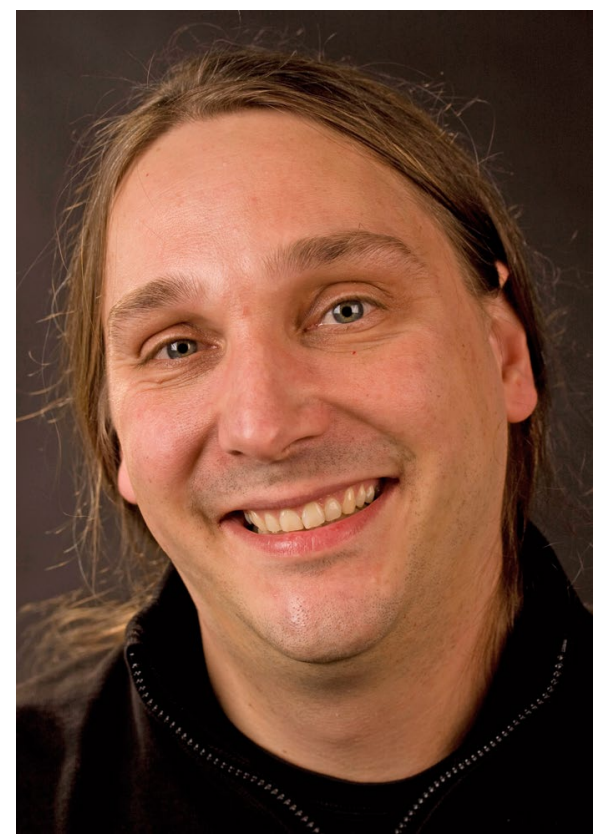

Credit: Mattias Trost

on what we could and should deliver was sparse. However, we slowly built a larger team of dedicated volunteers who took over specific tasks. It became obvious that the information flow from the central government and PHE was not optimal. Thus, many decisions were taken locally, and sometimes situations changed by the day.

We were very keen to build up a screening facility at Newcastle University. We had identified the perfect space: a large teaching lab next to a facility with biological safety level 3, where the patients' samples could be handled. However, there was opposition to this: some primary investigators told me that we would be responsible for deaths if we made mistakes. Some said the IT infrastructure and a laboratory information management system would be difficult to implement. And the NHS screening lab felt they could provide the required number of tests themselves. On the other hand, there were primary investigators telling me they could screen thousands of samples themselves and it was something undergraduates could do. This was the first time that I truly appreciated that managing senior academics is not an easy job.

As setting up our screening facility in the university received so much resistance, both internally and from some within the local NHS, we helped with what we could. We provided highly qualified staff to work within the NHS screening team, reagents, consumables, personal protective equipment, and instruments. As the NHS staff in the screening facility were not allowed to use public transportations, we set up a team of volunteers who drove them to and from work. This was very much appreciated and showed that small things could mean a lot to frontline staff.

As I am writing this, university staff members are validating alternative screening assays to increase resilience in case the supply chains for the current assay fail. A second group is generating hundreds of face shields using the university's 3D printing capabilities.

These are still the most rewarding moments in this crisis: seeing the ability of academics to utilize the resources at hand and their desire to do as much as one can in a moment of national crisis. Unlike medics, we academics do not have often the opportunity to have a direct impact on people's lives.

Now, almost 4 weeks after we wanted to start the screening facility, PHE has reached out, and it seems now more likely that we will set up a laboratory nonetheless. Although we regret that we encountered barriers to establishing this at an earlier point in the crisis, it will be a true pleasure to see more of this academic ingenuity providing a crucial service to those who need it most.

\section{Matthias Trost ${ }^{凶}$ \\ Newcastle University, Newcastle, UK.

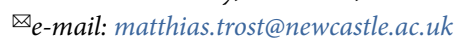

Published online: 11 May 2020 https://doi.org/10.1038/s41591-020-0885-5 